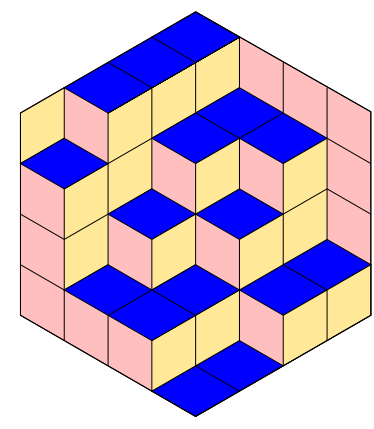

ALGEBRAIC COMBINATORICS

\author{
Akihide Hanaki \& Masayoshi Yoshikawa \\ A construction of pairs of non-commutative rank 8 association schemes from \\ non-symmetric rank 3 association schemes \\ Volume 4, issue 3 (2021), p. 533-540. \\ <http://alco.centre-mersenne.org/item/ALCO_2021__4_3_533_0>
}

(C) The journal and the authors, 2021.

Some rights reserved.

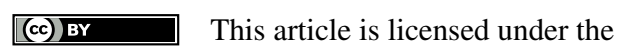

CREATIVE COMMONS ATTRIBUTION 4.0 INTERNATIONAL LiCENSE.

http://creativecommons.org/licenses/by/4.0/

Access to articles published by the journal Algebraic Combinatorics on the website http://alco.centre-mersenne.org/ implies agreement with the Terms of Use (http://alco.centre-mersenne.org/legal/).

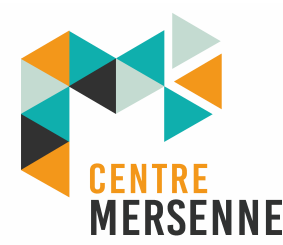

Algebraic Combinatorics is member of the Centre Mersenne for Open Scientific Publishing www.centre-mersenne.org 


\title{
A construction of pairs of non-commutative rank 8 association schemes from non-symmetric rank 3 association schemes
}

\author{
Akihide Hanaki \& Masayoshi Yoshikawa
}

\begin{abstract}
We construct a pair of non-commutative rank 8 association schemes from a rank 3 non-symmetric association scheme. For the pair, two association schemes have the same character table but different Frobenius-Schur indicators. This situation is similar to that for the dihedral and quaternion groups of order 8 . We also determine the structures of adjacency algebras of the rank 8 schemes over the rational number field.
\end{abstract}

\section{INTRODUCTION}

From a rank 3 non-symmetric association scheme of order $n-1$, we construct a pair of association schemes $(\mathcal{D}, \mathcal{Q})$ with the following properties.

- $\mathcal{D}$ and $\mathcal{Q}$ are non-commutative, of order $4 n$, and of rank 8.

- $\mathcal{D}$ and $\mathcal{Q}$ have the same character tables but their Frobenius-Schur indicators are different.

These properties are similar to the pair of the dihedral group $D_{8}$ and the quaternion group $Q_{8}$ of order 8 .

In the theory of association schemes, the adjacency algebras are mainly considered over the complex number field. In this paper, we determine the structures of adjacency algebras of $\mathcal{D}$ and $\mathcal{Q}$ over the rational number field $\mathbb{Q}$. We prove

$$
\begin{aligned}
& \mathbb{Q} \mathcal{D} \cong \mathbb{Q} \oplus \mathbb{Q} \oplus \mathbb{Q} \oplus \mathbb{Q} \oplus \mathrm{M}_{2}(\mathbb{Q}), \\
& \mathbb{Q} \mathcal{Q} \cong \mathbb{Q} \oplus \mathbb{Q} \oplus \mathbb{Q} \oplus \mathbb{Q} \oplus \mathbb{Q}(-1,-n+1),
\end{aligned}
$$

where $\mathrm{M}_{2}(\mathbb{Q})$ is the full matrix algebra of degree 2 and $\mathbb{Q}(-1,-n+1)$ is the quaternion division algebra.

It is known that a rank 3 non-symmetric association scheme of order $n-1$ exists if and only if there exists a skew-Hadamard matrix of order $n$ with $n \equiv 0(\bmod 4)$. There is a conjecture that such a matrix of order $n$ exists for an arbitrary $n \equiv 0$ $(\bmod 4)$.

\footnotetext{
Manuscript received 21st November 2019, revised and accepted 20th December 2020.
}

KEYwORDS. Character table, quaternion algebras, association schemes.

ACKNowledgements. Akihide Hanaki was supported by JSPS KAKENHI Grant Number JP17K05165. Masayoshi Yoshikawa was supported by JSPS KAKENHI Grant Number JP17K05173. 


\section{Preliminaries}

For a field $K$, we denote by $\mathrm{M}_{n}(K)$ the full matrix algebra of degree $n$ over $K$. For a matrix $M$, the transposed matrix of $M$ will be denoted by ${ }^{t} M$. By $I_{n}$, we denote the identity matrix of degree $n$. By $J_{n}$, we denote the $n \times n$ matrix with all entries 1.

We define an association scheme in matrix form. Let $\mathcal{S}=\left\{A_{0}, \ldots, A_{d}\right\}$ be a set of non-zero $n \times n$ matrices with entries in $\{0,1\}$. Then the set $\mathcal{S}$ is called an association scheme of order $n$ and rank $d+1$ if

(1) $A_{0}=I_{n}$,

(2) $\sum_{i=0}^{d} A_{i}=J_{n}$, and

(3) for any $0 \leqslant i, j \leqslant d,{ }^{t} A_{i}$ and $A_{i} A_{j}$ are linear combinations of $A_{0}, \ldots, A_{d}$.

By definition, all rows of $A_{i}$ contain the same number of 1 . We call this number the valency of $A_{i}$ and denote it by $n_{i}$. The association scheme $\mathcal{S}$ is said to be symmetric if ${ }^{t} A_{i}=A_{i}$ for all $0 \leqslant i \leqslant d$ and non-symmetric otherwise. The association scheme $\mathcal{S}$ is said to be commutative if $A_{i} A_{j}=A_{j} A_{i}$ for all $0 \leqslant i, j \leqslant d$ and non-commutative otherwise. For a field $K, K \mathcal{S}:=\bigoplus_{i=0}^{d} K A_{i}$ is a $(d+1)$-dimensional $K$-algebra by the condition (3). We call $K \mathcal{S}$ the adjacency algebra of $\mathcal{S}$ over $K$. It is known that $K \mathcal{S}$ is semisimple if the characteristic of $K$ is 0 [5, Theorem 4.1.3 (ii)]. A representation of $\mathcal{S}$ over $K$ means a $K$-algebra homomorphism from $K \mathcal{S}$ to a full matrix algebra $\mathrm{M}_{t}(K)$ for some positive integer $t$.

A subset $\mathcal{T}$ of an association scheme $\mathcal{S}$ is called a closed subset if $e_{\mathcal{T}}:=$ $n_{\mathcal{T}}^{-1} \sum_{A_{i} \in \mathcal{T}} A_{i}$ is an idempotent, where $n_{\mathcal{T}}:=\sum_{A_{i} \in \mathcal{T}} n_{i}$. A closed subset $\mathcal{T}$ of $\mathcal{S}$ is said to be normal if $e_{\mathcal{T}}$ is a central element of the complex adjacency algebra $\mathbb{C} \mathcal{S}$. A closed subset $\mathcal{T}$ of $\mathcal{S}$ is said to be strongly normal if ${ }^{t} A_{j} A_{i} A_{j} \in \bigoplus_{A_{\ell} \in \mathcal{T}} \mathbb{C} A_{\ell}$ for $A_{i} \in \mathcal{T}$ and $A_{j} \in \mathcal{S}$. It is known that a strongly normal closed subset is normal. The thin radical of $\mathcal{S}$ is $O_{\theta}(\mathcal{S})=\left\{A_{i} \mid n_{i}=1\right\}$ and is known to be a closed subset of $\mathcal{S}$. The thin residue $O^{\theta}(\mathcal{S})$ of $\mathcal{S}$ is the intersection of all strongly normal closed subsets of $\mathcal{S}$ and is known to be a strongly normal closed subset of $\mathcal{S}$. For details, the reader is referred to [5] or [2].

Now we consider the adjacency algebra over the complex number field $\mathbb{C}$. By Wedderburn's theorem $\left[4,3.5\right.$ Theorem], $\mathbb{C} \mathcal{S} \cong \bigoplus_{i=1}^{\ell} \mathrm{M}_{t_{i}}(\mathbb{C})$ for some $t_{1}, \ldots, t_{\ell}$. The set of projections $\mathbb{C S} \rightarrow \mathrm{M}_{t_{i}}(\mathbb{C})(i=1, \ldots, \ell)$ is a complete set of representatives of equivalence classes of irreducible representations of $\mathbb{C S}$. The matrix trace of a representation is called the character of the representation. By $\operatorname{Irr}(\mathcal{S})=\left\{\chi_{1}, \ldots, \chi_{\ell}\right\}$, we denote the set of irreducible characters of $\mathbb{C S}$. The $\ell \times(d+1)$ matrix $\left(\chi_{i}\left(A_{j}\right)\right)$ is called the character table of $\mathcal{S}$. Since $\mathbb{C S}$ is defined as a matrix algebra, the map $A_{i} \mapsto A_{i}$ is a representation. We call it the standard representation and its character the standard character of $\mathcal{S}$. The decomposition of the standard character into the irreducibles is written as $\sum_{\chi \in \operatorname{Irr}(\mathcal{S})} m_{\chi} \chi$. We call the coefficient $m_{\chi}$ the multiplicity of $\chi$.

The Frobenius-Schur indicator $\nu(\chi)$ of $\chi \in \operatorname{Irr}(\mathcal{S})$ is defined by

$$
\nu(\chi):=\frac{m_{\chi}}{n \chi\left(A_{0}\right)} \sum_{i=0}^{d} \frac{1}{n_{i}} \chi\left(A_{i}{ }^{2}\right) .
$$

An irreducible character is said to be of the first kind if it is afforded by a real representation, of the second kind if it is afforded by a representation which is equivalent to its complex conjugate but is not of the first kind, and of the third kind if it is not of the first or second kind. The following theorem is known. 
Theorem $2.1([3,(7,5),(7.6)])$.

(1) For $\chi \in \operatorname{Irr}(\mathcal{S})$,

$$
\nu(\chi)= \begin{cases}1 & \text { if } \chi \text { is of the first kind, } \\ -1 & \text { if } \chi \text { is of the second kind, } \\ 0 & \text { if } \chi \text { is of the third kind. }\end{cases}
$$

(2) $\sum_{\chi \in \operatorname{Irr}(\mathcal{S})} \nu(\chi) \chi\left(A_{0}\right)=\sharp\left\{A_{i} \in \mathcal{S} \mid{ }^{t} A_{i}=A_{i}\right\}$.

\section{Construction}

Let $\left\{A_{0}=I_{n-1}, A_{1}, A_{2}={ }^{t} A_{1}\right\}$ be a non-symmetric rank 3 association scheme of order $n-1$. Remark that $n \equiv 0(\bmod 4)$ for such an association scheme to exist. We will construct a pair $(\mathcal{D}, \mathcal{Q})$ of association schemes with the properties described in the Introduction.

Set $a:=n-1$ and $b:=(n-2) / 2$.

The following lemma is well-known.

\section{LEMma 3.1 .}

(1) $A_{1}^{2}=\frac{b-1}{2} A_{1}+\frac{b+1}{2} A_{2}$.

(2) $A_{2}^{2}=\frac{b+1}{2} A_{1}+\frac{b-1}{2} A_{2}$.

(3) $A_{1} A_{2}=A_{2} A_{1}=b A_{0}+\frac{b-1}{2} A_{1}+\frac{b-1}{2} A_{2}$.

Set $x:=(1,2,3,4), y:=(1,2)(3,4)$, permutations of degree 4 , and $G:=\langle x, y\rangle \cong D_{8}$. We identify the elements of $G$ with the corresponding permutation matrices, namely

$$
x=\left(\begin{array}{llll}
0 & 1 & 0 & 0 \\
0 & 0 & 1 & 0 \\
0 & 0 & 0 & 1 \\
1 & 0 & 0 & 0
\end{array}\right), \quad y=\left(\begin{array}{llll}
0 & 1 & 0 & 0 \\
1 & 0 & 0 & 0 \\
0 & 0 & 0 & 1 \\
0 & 0 & 1 & 0
\end{array}\right),
$$

and so on. The next lemma is clear by definition.

Lemma 3.2. As matrices, $1+x^{2}=x y+x^{3} y$.

We keep the above notations in the rest of this article.

3.1. The association scheme $\mathcal{D}$. We define $n \times n$ matrices by

$$
E:=\left(\begin{array}{c|c}
0 & 1 \ldots 1 \\
\hline 1 & \\
\vdots & O \\
1 &
\end{array}\right), \quad \tilde{A}_{i}:=\left(\begin{array}{c|c}
0 & 0 \ldots 0 \\
\hline 0 & \\
\vdots & A_{i} \\
0 &
\end{array}\right) \quad(i=1,2) .
$$

By Lemma 3.1 and direct calculations, we have the following lemma.

\section{LEMMA 3.3}

(1) $E^{2}+\tilde{A}_{1} \tilde{A}_{2}+\tilde{A}_{2} \tilde{A}_{1}=a I_{n}+b \tilde{A}_{1}+b \tilde{A}_{2}$.

(2) $\tilde{A}_{1}^{2}+\tilde{A}_{2}^{2}=b \tilde{A}_{1}+b \tilde{A}_{2}$.

(3) $E \tilde{A}_{1}+\tilde{A}_{2} E=E \tilde{A}_{2}+\tilde{A}_{1} E=b E$.

We consider a subgroup $H:=C_{G}(y)=\left\{1, x^{2}, y, x^{2} y\right\} \cong C_{2} \times C_{2}$ of $G$. It is easy to see that $\sum_{h \in H} h=\sum_{g \in G \backslash H} g=J_{4}$. We define $4 n \times 4 n$ matrices by

$$
\sigma_{h}:=I_{n} \otimes h \quad \text { for } h \in H
$$


and

$$
\begin{aligned}
\mu_{g} & :=E \otimes g+\tilde{A_{1}} \otimes g y+\tilde{A}_{2} \otimes g x^{2} y \\
& =\left(I_{n} \otimes g\right)\left(E \otimes 1+\tilde{A_{1}} \otimes y+\tilde{A}_{2} \otimes x^{2} y\right) \\
& =\left(E \otimes 1+\tilde{A_{1}} \otimes x^{2} y+\tilde{A}_{2} \otimes y\right)\left(I_{n} \otimes g\right) \quad \text { for } g \in G \backslash H .
\end{aligned}
$$

We will show that $\mathcal{D}:=\left\{\sigma_{h} \mid h \in H\right\} \cup\left\{\mu_{g} \mid g \in G \backslash H\right\}$ forms an association scheme.

LEMMA 3.4. The set $\mathcal{D}$ is closed under the transposition and

$$
\sum_{h \in H} \sigma_{h}+\sum_{g \in G \backslash H} \mu_{g}=J_{4 n} .
$$

Proof. We have ${ }^{t} \sigma_{h}=\sigma_{h}$ for $h \in H,{ }^{t} \mu_{x y}=\mu_{x y},{ }^{t} \mu_{x^{3} y}=\mu_{x^{3} y}$ and ${ }^{t} \mu_{x}=\mu_{x^{3}}$. Thus $\mathcal{D}$ is closed under the transposition.

Since $\sum_{h \in H} h=\sum_{g \in G \backslash H} g=J_{4}$ and $E+\tilde{A}_{1}+\tilde{A}_{2}=J_{n}-I_{n}$, we have $\sum_{h \in H} \sigma_{h}+$ $\sum_{g \in G \backslash H} \mu_{g}=J_{4 n}$.

\section{LEMMA 3.5 .}

(1) $\sigma_{h} \sigma_{h^{\prime}}=\sigma_{h h^{\prime}}$ for $h, h^{\prime} \in H$.

(2) $\sigma_{h} \mu_{g}=\mu_{h g}$ and $\mu_{g} \sigma_{h}=\mu_{g h}$ for $h \in H$ and $g \in G \backslash H$.

(3) $\mu_{g} \mu_{g^{\prime}}=a \sigma_{g g^{\prime}}+b \mu_{g g^{\prime} x}+b \mu_{g g^{\prime} x^{3}}$ for $g, g^{\prime} \in G \backslash H$.

Proof. It is easy to show that (1) and (2) hold.

Suppose $g, g^{\prime} \in G \backslash H$. Remark that $g g^{\prime} \in H$. By $g^{\prime} x^{2}=x^{2} g^{\prime}, y g^{\prime}=g^{\prime} x^{2} y$, Lemma 3.2 and Lemma 3.3, we have

$$
\begin{aligned}
\mu_{g} \mu_{g^{\prime}} & =\left(I_{n} \otimes g\right)\left(E \otimes 1+\tilde{A}_{1} \otimes y+\tilde{A}_{2} \otimes x^{2} y\right)\left(E \otimes 1+\tilde{A}_{1} \otimes x^{2} y+\tilde{A}_{2} \otimes y\right)\left(I_{n} \otimes g^{\prime}\right) \\
& =\left(I_{n} \otimes g\right)\left(a I_{n} \otimes 1+b\left(\tilde{A}_{1}+\tilde{A}_{2}\right) \otimes\left(1+x^{2}\right)+b E \otimes\left(y+x^{2} y\right)\right)\left(I_{n} \otimes g^{\prime}\right) \\
& =a \sigma_{g g^{\prime}}+b\left(\tilde{A}_{1}+\tilde{A}_{2}\right) \otimes g\left(1+x^{2}\right) g^{\prime}+b E \otimes g\left(y+x^{2} y\right) g^{\prime} \\
& =a \sigma_{g g^{\prime}}+b\left(\tilde{A}_{1}+\tilde{A}_{2}\right) \otimes g g^{\prime}\left(1+x^{2}\right)+b E \otimes g g^{\prime} x^{2}\left(y+x^{2} y\right) \\
& =a \sigma_{g g^{\prime}}+b\left(\tilde{A}_{1}+\tilde{A}_{2}\right) \otimes g g^{\prime}\left(x y+x^{3} y\right)+b E \otimes g g^{\prime} x^{2} y\left(x y+x^{3} y\right) \\
& =a \sigma_{g g^{\prime}}+b\left(\tilde{A}_{1}+\tilde{A}_{2}\right) \otimes g g^{\prime}\left(x y+x^{3} y\right)+b E \otimes g g^{\prime}\left(x+x^{3}\right) \\
& =a \sigma_{g g^{\prime}}+b \mu_{g g^{\prime} x}+b \mu_{g g^{\prime} x^{3}} .
\end{aligned}
$$

Now (3) holds.

THEOREM 3.6. The set $\mathcal{D}=\left\{\sigma_{h} \mid h \in H\right\} \cup\left\{\mu_{g} \mid g \in G \backslash H\right\}$ forms an association scheme.

Proof. This is clear by Lemma 3.4 and Lemma 3.5 .

We summarize basic properties of the association scheme $\mathcal{D}$ by Lemma 3.4 and Lemma 3.5.

Proposition 3.7. For the association scheme $\mathcal{D}=\left\{\sigma_{1}, \sigma_{x^{2}}, \sigma_{y}, \sigma_{x^{2} y}, \mu_{x}, \mu_{x^{3}}, \mu_{x y}, \mu_{x^{3} y}\right\}$, the following properties hold.

(1) The valencies of $\sigma_{1}, \sigma_{x^{2}}, \sigma_{y}, \sigma_{x^{2} y}$ are 1 and the valencies of $\mu_{x}, \mu_{x^{3}}, \mu_{x y}$, $\mu_{x^{3} y}$ are $n-1$.

(2) The thin radical is $O_{\theta}(\mathcal{D})=\left\{\sigma_{1}, \sigma_{x^{2}}, \sigma_{y}, \sigma_{x^{2} y}\right\}$, and is normal in $\mathcal{D}$.

(3) The thin residue is $O^{\theta}(\mathcal{D})=\left\{\sigma_{1}, \sigma_{x^{2}}, \mu_{x}, \mu_{x^{3}}\right\}$.

(4) The matrices $\sigma_{1}, \sigma_{x^{2}}, \sigma_{y}, \sigma_{x^{2} y}, \mu_{x y}, \mu_{x^{3} y}$ are symmetric and $\mu_{x}, \mu_{x^{3}}$ are non-symmetric. 
3.2. The Association scheme $\mathcal{Q}$. We define $n \times n$ matrices by

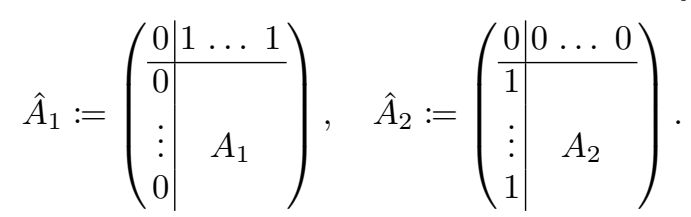

By Lemma 3.1 and direct calculations, we have the following lemma.

LEMMA 3.8.

(1) $\hat{A}_{1}^{2}+\hat{A}_{2}^{2}=b\left(J_{n}-I_{n}\right)$.

(2) $\hat{A}_{1} \hat{A}_{2}+\hat{A}_{2} \hat{A}_{1}=b J_{n}+(a-b) I_{n}$.

We consider a subgroup $K:=C_{G}(x)=\left\{1, x, x^{2}, x^{3}\right\} \cong C_{4}$ of $G$. It is easy to see that $\sum_{k \in K} k=\sum_{g \in G \backslash K} g=J_{4}$. We define $4 n \times 4 n$ matrices by

$$
\sigma_{k}:=I_{n} \otimes k \quad \text { for } k \in K
$$

and

$$
\begin{aligned}
\tau_{g} & :=\hat{A_{1}} \otimes g+\hat{A_{2}} \otimes g x^{2} \\
& =\left(I_{n} \otimes g\right)\left(\hat{A_{1}} \otimes 1+\hat{A_{2}} \otimes x^{2}\right) \\
& =\left(\hat{A_{1}} \otimes 1+\hat{A_{2}} \otimes x^{2}\right)\left(I_{n} \otimes g\right) \quad \text { for } g \in G \backslash K .
\end{aligned}
$$

We will show that $\mathcal{Q}:=\left\{\sigma_{k} \mid k \in K\right\} \cup\left\{\tau_{g} \mid g \in G \backslash K\right\}$ forms an association scheme.

LEMMA 3.9. The set $\mathcal{Q}$ is closed under the transposition and $\sum_{k \in K} \sigma_{k}+\sum_{g \in G \backslash K} \tau_{g}=$ $J_{4 n}$.

Proof. We have ${ }^{t} \sigma_{1}=\sigma_{1},{ }^{t} \sigma_{x^{2}}=\sigma_{x^{2}},{ }^{t} \sigma_{x}=\sigma_{x^{3}},{ }^{t} \tau_{y}=\tau_{x^{2} y}$, and ${ }^{t} \tau_{x y}=\tau_{x^{3} y}$. Thus $\mathcal{D}$ is closed under the transposition.

Since $\sum_{k \in K} k=\sum_{g \in G \backslash K} g=J_{4}$ and $I_{n}+\hat{A}_{1}+\hat{A}_{2}=J_{n}$, we have $\sum_{k \in K} \sigma_{k}+$ $\sum_{g \in G \backslash K} \tau_{g}=J_{4 n}$.

LEMMA 3.10 .

(1) $\sigma_{k} \sigma_{k^{\prime}}=\sigma_{k k^{\prime}}$ for $k, k^{\prime} \in K$.

(2) $\sigma_{k} \tau_{g}=\tau_{k g}$ and $\tau_{g} \sigma_{k}=\tau_{g k}$ for $k \in K$ and $g \in G \backslash K$.

(3) $\tau_{g} \tau_{g^{\prime}}=a \sigma_{g g^{\prime} x^{2}}+b \tau_{g g^{\prime} x y}+b \tau_{g g^{\prime} x^{3} y}$ for $g, g^{\prime} \in G \backslash K$.

Proof. It is easy to show that (1) and (2) hold.

Suppose $g, g^{\prime} \in G \backslash K$. Remark that $g g^{\prime} \in K$. By Lemma 3.2 and Lemma 3.8, we have

$$
\begin{aligned}
\tau_{g} \tau_{g^{\prime}} & =\left(I_{n} \otimes g\right)\left(\hat{A_{1}} \otimes 1+\hat{A_{2}} \otimes x^{2}\right)^{2}\left(I_{n} \otimes g^{\prime}\right) \\
& =\left(I_{n} \otimes g g^{\prime}\right)\left({\hat{A_{1}}}^{2} \otimes 1+\left(\hat{A_{1}} \hat{A}_{2}+\hat{A}_{2} \hat{A_{1}}\right) \otimes x^{2}+{\hat{A_{1}}}^{2} \otimes 1\right) \\
& =\left(I_{n} \otimes g g^{\prime}\right)\left(b\left(J_{n}-I_{n}\right) \otimes 1+\left(b J_{n}+(a-b) I_{n}\right) \otimes x^{2}\right) \\
& =\left(I_{n} \otimes g g^{\prime}\right)\left(a I_{n} \otimes x^{2}+b\left(J_{n}-I_{n}\right) \otimes\left(1+x^{2}\right)\right) \\
& =\left(I_{n} \otimes g g^{\prime}\right)\left(a I_{n} \otimes x^{2}+b\left(\hat{A_{1}}+\hat{A_{2}}\right) \otimes\left(1+x^{2}\right)\right) \\
& =\left(I_{n} \otimes g g^{\prime}\right)\left(a I_{n} \otimes x^{2}+b\left(\hat{A_{1}}+\hat{A_{2}}\right) \otimes\left(x y+x^{3} y\right)\right) \\
& =a \sigma_{g g^{\prime} x^{2}}+b \tau_{g g^{\prime} x y}+b \tau_{g g^{\prime} x^{3} y} .
\end{aligned}
$$

Now (3) holds.

TheOREM 3.11. The set $\mathcal{Q}=\left\{\sigma_{k} \mid k \in K\right\} \cup\left\{\tau_{g} \mid g \in G \backslash K\right\}$ forms an association scheme. 
Proof. This is clear by Lemma 3.9 and Lemma 3.10 .

We summarize basic properties of the association scheme $\mathcal{Q}$ by Lemma 3.9 and Lemma 3.10.

Proposition 3.12. For the association scheme $\mathcal{Q}=\left\{\sigma_{1}, \sigma_{x^{2}}, \sigma_{x}, \sigma_{x^{3}}, \tau_{x y}, \tau_{x^{3} y}, \tau_{y}, \tau_{x^{2} y}\right\}$, the following properties hold.

(1) The valencies of $\sigma_{1}, \sigma_{x^{2}}, \sigma_{x}, \sigma_{x^{3}}$ are 1 and the valencies of $\tau_{x y}, \tau_{x^{3} y}, \tau_{y}, \tau_{x^{2} y}$ are $n-1$.

(2) The thin radical is $O_{\theta}(\mathcal{Q})=\left\{\sigma_{1}, \sigma_{x^{2}}, \sigma_{x}, \sigma_{x^{3}}\right\}$, and is normal in $\mathcal{Q}$.

(3) The thin residue is $O^{\theta}(\mathcal{Q})=\left\{\sigma_{1}, \sigma_{x^{2}}, \tau_{x y}, \tau_{x^{3} y}\right\}$.

(4) The matrices $\sigma_{1}, \sigma_{x^{2}}$ are symmetric and $\sigma_{x}, \sigma_{x^{3}}, \tau_{x y}, \tau_{x^{3} y}, \tau_{y}, \tau_{x^{2} y}$ are nonsymmetric.

\section{The character tables of $\mathcal{D}$ and $\mathcal{Q}$}

In this section, we will determine the character tables of $\mathcal{D}$ and $\mathcal{Q}$. Consequently, we can see that the tables are the same. Moreover, we will show that their FrobeniusSchur indicators are different.

Proposition 4.1. The character table of $\mathcal{D}$ is

\begin{tabular}{c|cccccccc|c} 
& $\sigma_{1}$ & $\sigma_{x^{2}}$ & $\sigma_{y}$ & $\sigma_{x^{2} y}$ & $\mu_{x}$ & $\mu_{x^{3}}$ & $\mu_{x y}$ & $\mu_{x^{3} y}$ & $m_{\chi_{i}}$ \\
\hline$\chi_{1}$ & 1 & 1 & 1 & 1 & $n-1$ & $n-1$ & $n-1$ & $n-1$ & 1 \\
$\chi_{2}$ & 1 & 1 & -1 & -1 & $n-1$ & $n-1$ & $-n+1$ & $-n+1$ & 1 \\
$\chi_{3}$ & 1 & 1 & 1 & 1 & -1 & -1 & -1 & -1 & $n-1$ \\
$\chi_{4}$ & 1 & 1 & -1 & -1 & -1 & -1 & 1 & 1 & $n-1$ \\
$\chi_{5}$ & 2 & -2 & 0 & 0 & 0 & 0 & 0 & 0 & $n$
\end{tabular}

The Frobenius-Schur indicators are $\nu\left(\chi_{i}\right)=1(i=1,2,3,4,5)$.

Proof. By Proposition $3.7(2)$, the thin radical $O_{\theta}(\mathcal{D})=\left\{\sigma_{1}, \sigma_{x^{2}}, \sigma_{y}, \sigma_{x^{2} y}\right\}$ is a normal closed subset of $\mathcal{D}$. By [1, Theorem 3.5], we can determine $\chi_{1}$ and $\chi_{3}$. By Proposition $3.7(3)$, the thin residue $O^{\theta}(\mathcal{D})=\left\{\sigma_{1}, \sigma_{x^{2}}, \mu_{x}, \mu_{x^{3}}\right\}$ is a strongly normal closed subset of $\mathcal{D}$ and $\chi_{2}$ is determined. By [2, Theorem 3.5], $\chi_{4}:=\chi_{2} \chi_{3}$ is an irreducible character. Now, by $\sum_{i=1}^{5} m_{\chi_{i}} \chi_{i}(\rho)=0$ for $\sigma_{1} \neq \rho \in \mathcal{D}$ [5, Chap. 4], we can determine $\chi_{5}$.

Since $\chi_{i}(i=1,2,3,4)$ are rational characters of degree 1 , they are of the first kind. There are 6 symmetric matrices by Proposition 3.7 (4). By Theorem 2.1 (2),

$$
6=\sum_{i=1}^{5} \nu\left(\chi_{i}\right) \chi_{i}(1)=1+1+1+1+2 \nu\left(\chi_{5}\right)
$$

and we have $\nu\left(\chi_{5}\right)=1$.

Proposition 4.2. The character table of $\mathcal{Q}$ is

\begin{tabular}{c|cccccccc|c} 
& $\sigma_{1}$ & $\sigma_{x^{2}}$ & $\sigma_{x}$ & $\sigma_{x^{3}}$ & $\tau_{x y}$ & $\tau_{x^{3} y}$ & $\tau_{y}$ & $\tau_{x^{2} y}$ & $m_{\varphi_{i}}$ \\
\hline$\varphi_{1}$ & 1 & 1 & 1 & 1 & $n-1$ & $n-1$ & $n-1$ & $n-1$ & 1 \\
$\varphi_{2}$ & 1 & 1 & -1 & -1 & $n-1$ & $n-1$ & $-n+1$ & $-n+1$ & 1 \\
$\varphi_{3}$ & 1 & 1 & 1 & 1 & -1 & -1 & -1 & -1 & $n-1$ \\
$\varphi_{4}$ & 1 & 1 & -1 & -1 & -1 & -1 & 1 & 1 & $n-1$ \\
$\varphi_{5}$ & 2 & -2 & 0 & 0 & 0 & 0 & 0 & 0 & $n$
\end{tabular}

The Frobenius-Schur indicators are $\nu\left(\varphi_{i}\right)=1(i=1,2,3,4)$ and $\nu\left(\varphi_{5}\right)=-1$. 
Proof. By Proposition $3.12(2)$, the thin radical $O_{\theta}(\mathcal{Q})=\left\{\sigma_{1}, \sigma_{x^{2}}, \sigma_{x}, \sigma_{x^{3}}\right\}$ is a normal closed subset of $\mathcal{Q}$. We can determine $\varphi_{1}$ and $\varphi_{3}$. By Proposition $3.12(3)$, the thin residue $O^{\theta}(\mathcal{Q})=\left\{\sigma_{1}, \sigma_{x^{2}}, \tau_{x y}, \tau_{x^{3} y}\right\}$ is a strongly normal closed subset of $\mathcal{Q}$ and $\varphi_{2}$ is determined. Now the character table can be calculated in the same way as in Proposition 4.1.

Since $\varphi_{i}(i=1,2,3,4)$ are rational characters of degree 1 , they are of the first kind. There are 2 symmetric matrices by Proposition 3.12 (4). By Theorem 2.1 (2),

$$
2=\sum_{i=1}^{5} \nu\left(\varphi_{i}\right) \varphi_{i}(1)=1+1+1+1+2 \nu\left(\varphi_{5}\right)
$$

and we have $\nu\left(\varphi_{5}\right)=-1$.

\section{IRREDUCIBLE REPRESENTATIONS AND RATIONAL ADJACENCY ALGEBRAS}

We will determine irreducible representations and the structures of rational adjacency algebras of $\mathcal{D}$ and $\mathcal{Q}$, respectively.

Proposition 5.1. The map $T: \mathcal{D} \rightarrow \mathrm{M}_{2}(\mathbb{C})$ defined by

$$
\sigma_{x^{2}} \mapsto\left(\begin{array}{cc}
-1 & 0 \\
0 & -1
\end{array}\right), \quad \sigma_{y} \mapsto\left(\begin{array}{cc}
1 & 0 \\
0 & -1
\end{array}\right), \quad \mu_{x} \mapsto\left(\begin{array}{cc}
0 & -1 \\
n-1 & 0
\end{array}\right)
$$

is an irreducible representation of $\mathcal{D}$ affording $\chi_{5}$.

Proof. We can check all products in Lemma 3.5.

Similarly, we have the following proposition.

Proposition 5.2. The map $T^{\prime}: \mathcal{Q} \rightarrow \mathrm{M}_{2}(\mathbb{C})$ defined by

$$
\sigma_{x} \mapsto\left(\begin{array}{cc}
\sqrt{-1} & 0 \\
0 & -\sqrt{-1}
\end{array}\right), \quad \tau_{y} \mapsto\left(\begin{array}{cc}
0 & -1 \\
n-1 & 0
\end{array}\right)
$$

is an irreducible representation of $\mathcal{Q}$ affording $\varphi_{5}$.

Now we can determine the structures of rational adjacency algebras. To describe the result, we define (generalized) quaternion algebras [4, 1.6]. For a field $F$ and $r, s \in F \backslash\{0\}$, a quaternion algebra $F(r, s)$ is a four dimensional $F$-algebra with basis $1, \boldsymbol{i}, \boldsymbol{j}$, and $\boldsymbol{k}$ with the products

$$
\boldsymbol{i}^{2}=r, \quad \boldsymbol{j}^{2}=s, \quad \boldsymbol{i} \boldsymbol{j}=-\boldsymbol{j} \boldsymbol{i}=\boldsymbol{k} .
$$

If $r$ and $s$ are negative rational numbers, then $\mathbb{Q}(r, s)$ is a division algebra.

Proposition 5.3. As $\mathbb{Q}$-algebras, we have the following isomorphisms.

(1) $\mathbb{Q} \mathcal{D} \cong \mathbb{Q} \oplus \mathbb{Q} \oplus \mathbb{Q} \oplus \mathbb{Q} \oplus \mathrm{M}_{2}(\mathbb{Q})$.

(2) $\mathbb{Q} \mathcal{Q} \cong \mathbb{Q} \oplus \mathbb{Q} \oplus \mathbb{Q} \oplus \mathbb{Q} \oplus \mathbb{Q}(-1,-n+1) .(\mathbb{Q}(-1,-n+1)$ is a division algebra. $)$

Proof. Since $\chi_{i}(i=1,2,3,4)$ have rational values on $\mathcal{D}$, we can determine $\mathbb{Q} \oplus \mathbb{Q} \oplus \mathbb{Q} \oplus$ $\mathbb{Q}$. For the representation $T$ in Proposition 5.1 , it is easy to see that $T(\mathbb{Q D})=\mathrm{M}_{2}(\mathbb{Q})$. Thus (1) holds.

Since $\varphi_{i}(i=1,2,3,4)$ have rational values on $\mathcal{Q}$, we can determine $\mathbb{Q} \oplus \mathbb{Q} \oplus \mathbb{Q} \oplus \mathbb{Q}$. For the representation $T^{\prime}$ in Proposition 5.2, the set consisting of

$$
\begin{aligned}
T^{\prime}\left(\sigma_{1}\right) & =\left(\begin{array}{ll}
1 & 0 \\
0 & 1
\end{array}\right), & T^{\prime}\left(\sigma_{x}\right) & =\left(\begin{array}{cc}
\sqrt{-1} & 0 \\
0 & -\sqrt{-1}
\end{array}\right), \\
T^{\prime}\left(\tau_{y}\right) & =\left(\begin{array}{cc}
0 & -1 \\
n-1 & 0
\end{array}\right), & T^{\prime}\left(\tau_{x y}\right) & =\left(\begin{array}{cc}
0 & -\sqrt{-1} \\
-(n-1) \sqrt{-1} & 0
\end{array}\right)
\end{aligned}
$$


is a $\mathbb{Q}$-basis of $T^{\prime}(\mathbb{Q} \mathcal{Q})$. We can see that

$$
\begin{gathered}
T^{\prime}\left(\sigma_{x}\right)^{2}=-T^{\prime}\left(\sigma_{1}\right), \quad T^{\prime}\left(\tau_{y}\right)^{2}=-(n-1) T^{\prime}\left(\sigma_{1}\right), \\
T^{\prime}\left(\sigma_{x}\right) T^{\prime}\left(\tau_{y}\right)=-T^{\prime}\left(\tau_{y}\right) T^{\prime}\left(\sigma_{x}\right)=T^{\prime}\left(\tau_{x y}\right) .
\end{gathered}
$$

This shows that $T^{\prime}(\mathbb{Q} \mathcal{Q}) \cong \mathbb{Q}(-1,-n+1)$ and (2) holds.

\section{REMARK}

Our pair $(\mathcal{D}, \mathcal{Q})$ has similar properties to the pair $\left(D_{8}, Q_{8}\right)$, the dihedral and quaternion groups of order 8. Our association schemes have order $4 n$ and $n \equiv 0(\bmod 4)$, and thus $\left(D_{8}, Q_{8}\right)$ is not obtained by our construction. If we set $n=2$ and $A_{1}=A_{2}=O$ and apply our construction, then we can construct the pair $\left(D_{8}, Q_{8}\right)$ and all arguments are valid for it.

Acknowledgements. The authors would like to thank the anonymous referees for their helpful comments.

\section{REFERENCES}

[1] Akihide Hanaki, Representations of association schemes and their factor schemes, Graphs Combin. 19 (2003), no. 2, 195-201.

[2] _ Character products of association schemes, J. Algebra 283 (2005), no. 2, 596-603.

[3] Donald G. Higman, Coherent configurations. I. Ordinary representation theory, Geometriae Dedicata 4 (1975), no. 1, 1-32.

[4] Richard S. Pierce, Associative algebras, Graduate Texts in Mathematics, vol. 88, Springer-Verlag, New York-Berlin, 1982, Studies in the History of Modern Science, 9.

[5] Paul-Hermann Zieschang, An algebraic approach to association schemes, Lecture Notes in Mathematics, vol. 1628, Springer-Verlag, Berlin, 1996.

Akinide Hanaki, Faculty of Science, Shinshu University, 3-1-1 Asahi, Matsumoto, 390-8621, Japan E-mail : hanaki@shinshu-u.ac.jp

Url : http://math.shinshu-u.ac.jp/ hanaki

Masayoshi Yoshikawa, Department of Mathematics, Hyogo University of Teacher Education, 9421 Shimokume, Kato, Hyogo, 673-1494, Japan

E-mail : myoshi@hyogo-u.ac.jp 